\title{
Outdoor Lighting Ordinances: Tools to Preserve the Night Sky ${ }^{1}$
}

\author{
Donald R. Davis \\ International Dark-Sky Association, 3225 N. First Avenue, Tucson, \\ Arizona 85719 USA
}

\begin{abstract}
The dark night sky is rapidly being lost throughout the world due to wasted outdoor night lighting. Following warnings by astronomers ${ }^{1}$ and environmentalists, many communities are acting to preserve the night sky by enacting outdoor lighting codes. Here we briefly recount the history of codes and outline the essential elements of an effective code. Additional information, including a handbook on how to write a good outdoor lighting ordinance, is available from the International Dark-Sky Association web site, www.ida.org .
\end{abstract}

\section{Introduction}

For much of the 19th century the major astronomical observatories of the world, principally in Europe, were located close to major population centres - Paris, Berlin, London - where most astronomers lived and worked. The increasing quality of astronomical instrumentation coupled with the declining observational environment near major population centres led to the search for prime observing sites removed from cities. About a century ago sites such as Williams Bay, Wisconsin (Yerkes Observatory), Mt. Wilson and Mt. Hamilton (Lick Observatory) in California were identified and became major astronomical facilities.

Growth near observatories was relentless, however, particularly in the US West. Mt. Wilson was the first to experience significant adverse effects on its night sky quality due to explosive growth in the Los Angeles area. As shown in Figure 1, the Mt. Wilson sky brightness is calculated to have increased at the rate of nearly $0.3 \mathrm{mag}$ per decade from 1910 to 1970 . Owing to the loss of dark skies (although the Mt. Wilson seeing is still exceptional), deep sky observing terminated there in the early $1980 \mathrm{~s}^{2}$.

In the 1950 s and early 1960 s, other major astronomical sites in the US - Mt. Palomar, Kitt Peak, McDonald Observatory - were thought to be too remote from major cities to be significantly affected by light pollution. For example, Meinel (1960) wrote, "For the foreseeable future there appears to be

\footnotetext{
${ }^{1}$ This paper is dedicated to Dr. Arthur Hoag (1921-1999), whose pioneering efforts led to the first comprehensive outdoor lighting code.

${ }^{2}$ Technological advances combined with the exceptional seeing at Mt. Wilson enable outstanding astronomical research to still be done from this site on moderately bright (by astronomical standards) objects.
} 




SOUREes :A. SNhDAge, R. Garstane, J. Kristian. P. Nisensonet al., A. VAUGHAN

Figure 1. Sky brightness at Mt. Wilson observatory. Comparison of model predictions based on Garstang (1989) with measurements (data provided by S. Baliunas, private communication).

no serious threat to astronomical conditions on Kitt Peak ...". Within a decade, though, the light pollution threat to many major observatories was apparent to all. Riegel (1973) summarized the problem that outdoor lighting posed to astronomy. Hoag and Peterson (1974) expanded on the light pollution issue and described methods for ameliorating it. In an amazingly short time, explosive population growth in the southwestern US and its concomitant light pollution threatened the observatories' world-class dark skies.

Responding to this threat, astronomers from Kitt Peak National Observatory (KPNO) and the University of Arizona led an effort to establish a comprehensive outdoor lighting code (OLC) in Tucson and Pima County, the first such ordinance in the world. Following the adoption of this code in 1973, a permanent Outdoor Lighting Code Committee composed of representatives from the astronomical community, lighting engineers, general business people, electrical company representatives, licensed contractors and building engineers was created to address ongoing light pollution issues. Other municipalities near astronomical facilities, such as Flagstaff, Arizona, rapidly followed suit; Table 1 summarizes the status of OLCs currently in effect to protect major observatories.

While preserving dark skies was initially an issue for astronomers, in recent years it has become a concern to a much larger number of people. The awareness of light pollution as an environmental issue increased as more and more people were deprived of cherished views of the night sky - the Milky Way is disappearing into the milky haze of light pollution. Interestingly, though, in the US the solution to the light pollution environmental problem has been to continue adoption of local ordinances instead of addressing the issue at the national level 
through the Environmental Protection Agency. Local codes have proliferated and there are now more than 150 in effect in the United States, including four state-wide codes. Numerous others exist worldwide and the number is increasing rapidly as people are beginning to protect their night sky.

In some countries, a comprehensive approach to the light pollution problems may be feasible on the national level. Australia has a national standard for "The Control of Obtrusive Outdoor Lighting." This standard is the first comprehensive approach to containing detrimental effects of poor lighting practices. Countries with a strong environmental awareness on the national level may find that national legislation is the most useful tool for effectively combating light pollution.

\section{Table 1. Status of Outdoor Lighting Codes (OLC) for Protection of Selected Major Astronomical Observatories}

Observatory

KPNO, Kitt Peak, Arizona,

FLWO, Mt. Hopkins, Arizona

USNO, Lowell Observatory, Flagstaff, Arizona

Mt. Wilson Observatory,

Los Angeles, California

Palomar Observatory,

San Diego, California

Lick Observatory,

San Jose, California

Mauna Kea, Hawaii

Siding Spring Observatory,

Australia

McDonald Observatory,

Austin, Texas

Cerro Tololo Observatory,

La Serena, Chile

Roque de los Muchachos

Observatory, Canary Islands, Spain
Date of

First OLC Current OLC
Notes

1972

1999

a

1973

1989

$\mathrm{b}$

C

1984

1986

1980

1983

1974

1988

1988

1988

d

1999

1999

1999

1999

e

1988

1988

\footnotetext{
${ }^{a}$ Significant changes in 1999 code.

${ }^{b}$ First use of lumens cap; LPS near observatories.

${ }^{c}$ No comprehensive OLC; the city of Los Angeles is switching its street lighting to full cutoff.

${ }^{d}$ This code is currently undergoing revisions.

${ }^{e}$ First "norm" or regulation passed this year, previously only recommendations were passed.

${ }^{f}$ Code applies to the islands of La Palma and Tenerife.
} 


\section{How to Write an Outdoor Lighting Code}

To provide guidance for anyone wishing to create an OLC, the International Dark-Sky Association has developed an OLC Model Code Handbook, which is available on the IDA web site (www.darksky.org). This handbook provides information such as how to write a good code, protection of astronomical sites and discussion of the different types of illumination sources, with pros and cons for different applications. General guidelines for protecting the night sky through good lighting practices are also given by the Commission Internationale de l'Eclairage (CIE) 1997 technical report entitled "Guidelines for Minimizing Sky Glow."

A good outdoor lighting code includes the following elements:

- Minimize direct uplight. Light emitted above the horizontal plane directly contributes to light pollution, so outdoor luminaires should be full cutoff fixtures that emit no light above the horizontal plane.

- Use only the amount of light that is needed. Limits on the amount of light generated appropriate to the activities of the area are essential to controlling the overlighting situation that develops when outdoor lighting is used to attract people's attention (the "moth to the brightest candle" syndrome). Examples of lighting limits are given in the IDA Model Code Handbook.

- Curfews for unneeded light. Turning off lights when they are not needed significantly reduces light pollution (as well as saving energy) for much of the night. For example, turn off parking lot lights (except for needed security lighting) after business hours.

- Use low pressure sodium (LPS) lighting near astronomical observatories. LPS is the astronomer's choice for outdoor lighting since the illumination is only at one color, a golden yellow, and can readily be filtered out for many astronomical applications (see the article by Luginbuhl in this volume). A bonus is that LPS is one of the most efficient lighting sources available: it produces about eight times as much light for each watt of electrical energy consumed as does an incandescent lamp.

Preserving dark skies through outdoor lighting ordinances does not end with the passing of an ordinance - effective enforcement is crucial in order to reap benefits. Enforcement includes: (a) ensuring that plans for development projects comply with the code, and (b) field inspection for compliance once the lighting is installed. Obviously it is much better to recognize and fix non-compliant cases in the first step rather than the second one. The first and most critical step in enforcement comes in the design/plans review phase of development that requires outdoor lighting. Code compliance should be achieved through a knowledgeable lighting engineer and verified by an appropriate jurisdictional authority. Next, the actual installation must be inspected to ensure that what has been installed complies with the approved plan. Finally, a mechanism should be established for surveying the community to identify non-compliant lighting. 
It should be noted that most non-compliant lighting is installed out of ignorance of the outdoor lighting code.

There can be various procedural difficulties in implementing an OLC. First, there may be multiple jurisdictions in a geographic area that need to adopt similar codes in order to protect dark skies. For example, many urban areas in the US are a patchwork of small communities: there are six "jurisdictional authorities" within Pima County, Arizona, where KPNO and the Whipple Observatory are located. Without effective enforcement and public education programmes, however, an outdoor lighting code is just another arcane example of bureaucratic rubbish.

The bedrock of code compliance is education: all segments of the population must be aware of the code, its purpose and how to comply with it. This education process includes training sessions for lighting professionals, public officials and developers, newspaper articles and public talks. Brochures summarizing key points of good lighting for distribution to the general public are also helpful. Finally, all optical observatories should have a knowledgeable staff member charged with responsibility for light pollution issues. Most such institutions will not be as fortunate as La Palma, which has a full-time staff for the "Oficina Tecnica por la Proteccion de la Calidad del Cielo," but the function of dark sky preservation should be a structured staff activity at all observatories. Resources sufficient to accomplish the task must be allocated, ideally as a line item in the observatory budget. Cerro Tololo, for example, is now committing around $1 \%$ of its resources to light pollution issues (M. Smith, private communication).

\section{Do Lighting Codes Work?}

How effective are outdoor lighting codes in protecting observatories? The answer to this question has not yet been quantified. Despite the call over a quarter of a century ago for systematic monitoring programmes at major astronomical observatories (Riegel 1973), no such programme has, to my knowledge, been implemented. The best datum is from Pilachowski et al. (1989), who measured the KPNO sky brightness near solar minimum (Figure 2). They found a zenith $V$-brightness of $\sim 21.9 \mathrm{mag} \operatorname{arcsec}^{-2}$, which was 0.04 magnitudes fainter than sky brightness predictions (Garstang 1989). They attributed the difference to the efficiency of the Tucson/Pima County Outdoor Lighting Code. Unfortunately, these measurements were not repeated at the 1996-97 solar minimum. Also shown in Figure 2 are sky brightness measurements from Mt. Hopkins (FLWO) and La Palma indicating that skies are still quite dark at those sites, despite significant population growth nearby. Lighting ordinances do seem to be protecting dark skies.

\section{Summary}

All major observatories should establish a continuing programme to measure sky brightness at their site. Such data are crucial, not only for knowing their effective limiting magnitude for scientific programmes, but also for establishing long-term sky brightness trends and for assessing how well outdoor lighting codes are doing their job of protecting dark skies. 




Figure 2. Zenith sky brightness in the $V$-band for La Palma, Kitt Peak National Observatory (KPNO) and the F. L. Whipple Observatory (FLWO) on Mt. Hopkins, Arizona.

The awareness of light pollution has just begun - it is comparable to the awakening to the problems of air and water pollution in the early 1960s. While light pollution does not pose the clear and present danger of air and water pollution, it does represent a more subtle threat to humanity. By cutting people off from their direct views of the stunning night sky, light pollution robs us of an awareness of the vast universe and the sense of awe and humility that it inspires. We have a heritage of connection to the heavens, a vital component of the natural world. As our dark skies disappear, we lose our appreciation of the historical significance that stars and planets have played in the development of our culture as navigation aids, as challenges to our sciences and as inspiration to our religions. Without night skies humanity is losing a critical factor in making us what we are today. This cannot be allowed to happen.

\section{References}

Garstang, R. H. 1989, Ann. Rev. Astr. Ap., 27, 19

Hoag, A. A., and Peterson, A. A. 1974, Kitt Peak National Observatory, April 1974

Meinel, A. B. 1960, In Stars and Stellar Systems, Vol.I, 154-175 [Editor: p.175 is quoted] Pilachowski, C. A., Africano, J. L., Goodrich, B. D., and Binkert,W. S. 1989, PASP, 101, 707

Riegel, K. W. 1973, Science, 179, 1285 